\title{
Starspots and spin-orbit alignment in the WASP-4 exoplanetary system
}

\author{
Roberto Sanchis-Ojeda ${ }^{1}$, Joshua N. Winn ${ }^{1}$, Matthew J. Holman ${ }^{2}$, \\ Joshua A. Carter ${ }^{1,2}$, David J. Osip ${ }^{3}$ and Cesar I. Fuentes ${ }^{2,4}$ \\ ${ }^{1}$ Department of Physics, and Kavli Institute for Astrophysics and Space Research, \\ Massachusetts Institute of Technology, Cambridge, MA 02139, USA \\ e-mail: rsanchis@MIT.EDU \\ ${ }^{2}$ Harvard-Smithsonian Center for Astrophysics, 60 Garden Street, Cambridge, MA 02138, USA \\ ${ }^{3}$ Las Campanas Observatory, Carnegie Observatories, Casilla 601, La Serena, Chile \\ ${ }^{4}$ Department of Physics and Astronomy, Northern Arizona University, PO Box 6010, Flagstaff, \\ AZ 86011
}

\begin{abstract}
We present the photometric analysis of 4 transits of the exoplanet WASP-4b, obtained with the Baade $6.5 \mathrm{~m}$ telescope, one of the two Magellan telescopes at Las Campanas. The light curves have a photometric precision of $0.5 \mathrm{mmag}$ and a time sampling of $30 \mathrm{~s}$. This high precision has allowed us to detect several "spot anomalies": temporary brightenings due to the occultation of a starspot on the transit chord. By analyzing these anomalies we find the sky-projected stellar obliquity to be $\lambda=1^{\circ}{ }_{-14}^{+12^{\circ}}$. The small value suggests that the planet migration mechanism preserved the initially low obliquity, or that tidal evolution has realigned the system.
\end{abstract}

Keywords. stars: spots, planetary systems, techniques: photometric

Until now, in the study of transiting planets, starspots have been a nuisance, a factor that limits the precision of our measurements. Most recently, different groups have been able to infer the rotational period of the host star due to the appearance of spots (Dittmann et al. 2009; Silva-Valio 2008; Silva-Valio et al. 2010). In our project (SanchisOjeda et al. 2010, in preparation) we report the observation of four different transits of WASP-4b (Wilson et al. 2008), in August and September of 2009 (see Fig. 1). In the figure we have identified two anomalies in the last two transits, which we interpret as the planet crossing in front of the same spot during two different transits. The same sort of spot anomalies can be observed in light curves by Southworth et al. (2009). The recurrence of the anomaly at a later phase of the transit favors the configuration where the orbital angular momentum and the axis of rotation of the star are aligned, as in this case, the trajectories of the spot on the surface and the planet would be almost parallel. In this project we quantify this statement, obtaining a value of the sky projected spin-orbit angle of the system with a simple geometric model.

The model only uses the times when the spot events occurred. The parameters are the periods of rotation of the two spots (ours and Southworth's) and the two angles that describe the orientation of the rotation of the star in the sky. In this model, we assume that the spots rotate at a fixed latitude on the surface of the star, with initial conditions fixed by the position of the center of the planet's shadow when the first observation of the spot ocurred. The period of rotation of that particular spot, and the angles $\lambda$ and $i_{s}$, determine completely the position of the spot at any other time. We define a $\chi^{2}$ function that penalizes models that fail to produce the second anomaly at the appropriate time, or that do produce anomalies during transits when none were observed. We also use the 


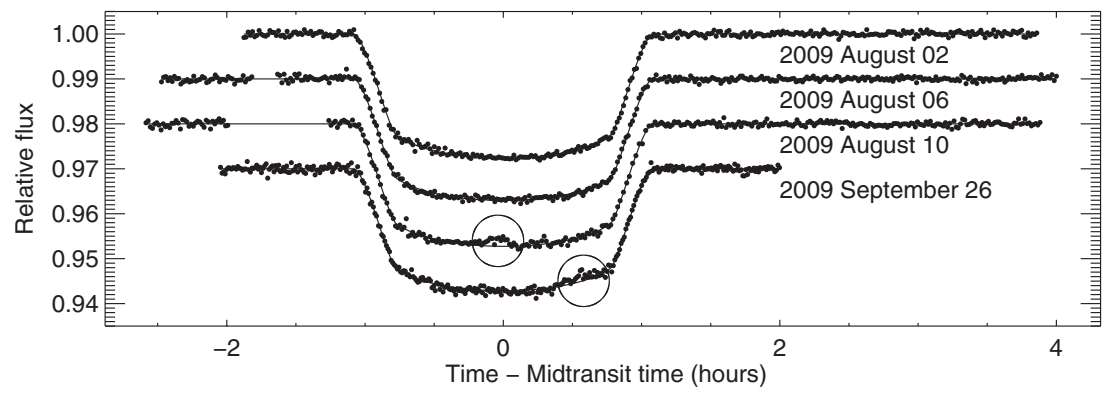

Figure 1. Four transits of WASP-4b, with two probable spot-crossing events highlighted with black circles.
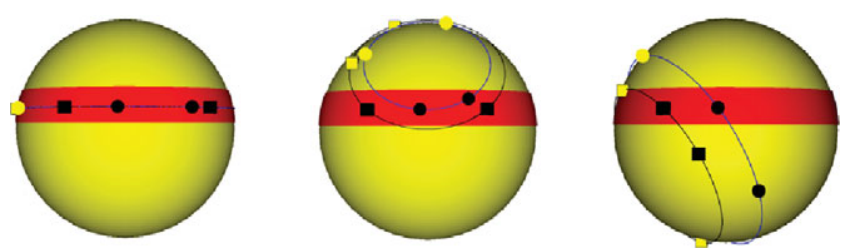

Figure 2. Circles (squares) represent the position of the spot during our (Southworth's) observed transits. Dark marks represent detections whereas light marks represent non-detections. The red dark band represents the transit chord. Left: $\lambda=0, i_{s}=90^{\circ}$, aligned case, allowed. Center: $\lambda=0, i_{s}=45^{\circ}$ case, still allowed. Right: $\lambda=45^{\circ}, i_{s}=90^{\circ}$ case, not allowed.

constraints $v \sin i_{S}=2.14 \pm 0.37 \mathrm{~km} / \mathrm{s}$ and $|\lambda|<90^{\circ}$ from the study of the RossiterMcLaughlin effect by Triaud et al. (2010).

Four different local minima of $\chi^{2}$ were found, in which the aligned case $\left(\lambda=0, i_{s}=90^{\circ}\right)$ is found to be the global minimum. The different allowed cases have very different periods for the spots, and also very different values for $i_{s}$, but they all give small values for $\lambda$. Fig. 2 shows geometrically why changing $i_{s}$ is not forbidden, whereas large values of $\lambda$ are ruled out. Running an MCMC algorithm to explore all possible minima, we are able to obtain a posteriori probability distribution for $\lambda$, obtaining a result of $\lambda=1^{\circ}{ }_{-14^{\circ}}^{\circ}$.

This method is a new powerful tool to measure the spin-orbit alignment of exoplanetary systems. It works best where the Rossiter-McLaughlin effect does not work, that is, for slowly rotating stars. The precision of the method can be affected by the unknown evolution of the spot, but the results would be highly improved if we could have more consecutive transits, making it possible to constrain also $i_{s}$. This is exactly what one will be able to get with the Kepler satellite, which is observing 170,000 stars continuously for 3.5 years. Future research will move in this direction, trying to apply this method to other exoplanetary systems in the Kepler field of view and improving the method at the same time.

\section{References}

Dittmann, J. A., Close, L. M., Green, E. M., \& Fenwick, M. 2009, ApJ, 701, 756

Silva-Valio, A. 2008, ApJ, 683, L179

Silva-Valio, A., Lanza, A. F., Alonso, R., \& Barge, P. 2010, A\&A, 510, A25

Southworth, J., et al. 2009, MNRAS, 399, 287

Triaud, A. H. M. J., et al. 2010, A\&A, 524, A25

Wilson, D. M., et al. 2008, ApJ, 675, L113 